\title{
Research, Practice and Theory in Didactics of Mathematics: towards Dialogue between Different Fields
}

\author{
Maria G. Bartolini Bussi \& Luciana Bazzini \\ $\underline{\text { bartolini@unimo.it_uciana.bazzini@unito.it }}$
}

\begin{abstract}
Acknowledging the complex relationships which the field of didactics of mathematics has with other research fields (e.g. mathematics, educational sciences, epistemology, history, psychology, semiotics, sociology, cognitive science), the authors analyze in this paper some cases of fruitful and of some failed dialogue between experts of the different fields. They discuss results of these dialogues, drawing on research studies carried out by the authors, within the paradigm of the Italian research in Mathematics Education
\end{abstract}

\section{Introduction}

In recent decades, several authors have emphasized the complex systemic nature of Didactics of Mathematics as a research field and its interrelations with different domains, like, for example, mathematics; educational sciences, epistemology; history; psychology; semiotics; sociology. (Steiner 1985, Biehler, Scholz, Straesser \& Winkelmann 1994, Wittmann, 1995, Godino \& Batanero 1997).

In particular, the last authors suggest distinguishing between 'Didactics of Mathematics' defined as 'the scientific and scholarly field of research which aims to identify, characterize, and understand the phenomena and processes conditioning the teaching and learning of mathematics'

and 'Mathematics Education' defined as

'the complex and heterogeneous social system which includes theory, development, and practice concerning the teaching and learning of mathematics'.

According to this definition, Mathematics Education includes Didactics of Mathematics as a subsystem. Such a distinction is fruitful because it highlights some distinguishing features of theoretical research in Didactics of Mathematics and their influence on Mathematics Education.

It is trivial to say that researchers in didactics of mathematics are interested mostly in those particular studies which are explicitly related to mathematics or to mathematics education, e. g.: epistemology of mathematics; history of mathematics / of mathematics education; psychology of mathematics / of mathematics education, sociology of mathematics / of mathematics education.

However, each of the above fields has its own scholars and specialists, who are very busy with their own research problems and their own methodologies. A fruitful dialogue between them and researchers in Mathematics Education is not easy. In the following, we analyse some cases, 
symmetrical or not. In particular, the interaction with historians of mathematics and with mathematicians will be taken into account.

Subsequently, we focus on two paradigmatic examples in which the interaction of different components (theoretical research and classroom practice) is a basic issue. The two reasearch studies deal with theoretical thinking in Geometry and on algebraic thinking respectively; they can also be reconsidered as cases within a specific research paradigm which is typical in the Italian tradition.

\section{A First Case: History and Didactics of Mathematics}

Historians of mathematics are a very well structured corporation, that is part of the corporation of historians of science. They have their own magazines and journals (e.g. Archive for the history of Exact Science, Historia Mathematica, just to quote a couple of them), research meetings and international conferences. National as well as international groups have emerged in the past years to try to establish a connection between historians and didacticians: e. g. the Commission INTERIREM "Épistemologie et Histoire des Mathématiques" in France ( Inter-IREM, 1997; 1998) and the International Study Group on the Relations between History and Pedagogy of Mathematics (HPM), which publishes a Newsletter regularly. Many conferences have been organized occasionally (see for example Jahnke, Knoche \& Otte 1996) or on a regular basis, e. g. the European Summer Universities on the History and the Epistemology in Mathematics Education.(see Lalande et al., eds., 1993 and Lagarto et al. Eds., 1996, and Radelet, 1999). An entire ICMI Study (1998) has been devoted to 'The role of the history of mathematics in the teaching and learning of mathematics' in Marseille - Luminy; furthermore a satellite meeting of ICME 9 dealt with the role of History in Mathematics Education (Horng and Lin, 2000). If we go through the list of contents of the volume

bringing together the work of the quoted ICMI Study (Fauvel \& van Maanen, 2000), we find many interesting issues, such as, the role of historical analysis in predicting and interpreting students' difficulties in mathematics and the relevance of historical studies in designing and analysing classroom activities.

These materials and the experiments carried out all over the world make it possible to go further in the discussion about the role of the history of mathematics in mathematics teaching.

An interesting question is: "Is peer cooperation between historians and didacticians possible?" 
Let us start from the position taken by Pepe (1990), an historian of mathematics, who wrote:

'Today the issue of the relationship between history and didactics of mathematics may be put in a very effective way, more effective than in the past: they are autonomous from each other and may cooperate to the development and to the diffusion of mathematical culture.'

Yet, the author insists that the meeting between history and didactics of mathematics may cause negative effects on each other. On the one hand, the introduction of history of mathematics into didactics of mathematics may produce risks for teaching, e. g.:

'the increase of notions to be learnt, with curious and interesting, yet not fundamental references; the loss of perspective, through the difficult and sometimes contradictory path of mathematical research in the past; the loss of certainty, with a wide diffusion of doubt in the everyday teaching'

On the other hand, the development of historical studies driven by didactical aims may clash with the good methodological rules of historical research:

'a teleological view may prevail, twisting the facts categories not related to the critical reconstruction of the past may be introduced not original studies may be developed, according to the didactical fashion'.

Finally, in the rest of the paper, the author discusses some examples of positive interaction between history and didactics of mathematics, mainly related to the introduction into teaching practice of the so-called applications of mathematics (astronomy, carthography and so on).

The author, an historian, in spite of the claimed 'cooperation' between them, deals with the relationships between history and didactics in a deeply asymmetrical way. The two lists of risks are only superficially symmetrical. Both didacticians and historians are running some risks, but the former have, at least, the hope to gain advantage for their discipline, whilst the latter are only to be be diverted from the accepted rules of historical research.

In symmetrical relationships both partners are expected to run some risks but to gain some advantages. Yet some historians seem to be convinced that no scientific advantage comes from their interaction with didacticians. Most historians are nice listeners and provocative critics of lectures where some interesting application of history of mathematics is presented, but they do not consider the instruments of analysis and the results of the didactical research useful in their own research studies. We think that, on the contrary, also historians might take advantage of some interaction with didacticians, at least when didacticians are making an epistemological analysis of the genesis of some concept, driven by the needs of interpreting students' difficulties and of planning effective classroom activities.

A large quotation from Jahnke et al. (1996) is useful:

History of mathematics is considered by many as fundamental research, and integrating history into teaching 
idea is misleading. The significance of history lies in its contribution to the general culture. Even more than for general history, it is true for history of science that the fundamental relation to culture is bounded to what is termed "Bildung" in Germany. If this is accepted, the immediate consequence is that we cannot live any longer with a situation in which mathematics educators have to fumble for subject matter which just might be adequate for teaching uses. Instead, the questions and viewpoints resulting from teaching must become an integral part of the methodology of historical research. Although many historians may fear that this could impose limitations on their own work, the opposite is true. Education will raise a host of questions which will substantially enrich historical research (Jahnke et al. (1996, p. X-XI).

With this motivation, the quoted authors organized a Conference in Essen (1992), where historians and didacticians met to present their own contributions. The dialogue was continued in the History and Pedagogy of Mathematics Group. Starting from the basic issue that the use of history in teaching mathematics involves different characters: researchers in history, researchers in education, teachers, students, Furinghetti (1997) tries an analysis of the different roles. In her view, the role of historians would be mainly of interpreting and mediating sources; some of them who are sensible of pedagogical problems point out specific situations suitable to a didactic transposition. Sometime the position of historians seems a bit 'integralist' and their intention to be 'politically correct' with regard to history is perceived by the world of education as a hidden warning to keep away from history. On the other hand it is true that in some cases the world of educators felt into the pitfall already met in the early attempts of introducing the computer in mathematics teaching: history and the computer are both appealing and appear good solutions of the problem of involving students in mathematics, thus the transposition in classroom was carried out mainly relying on the glamour and neglecting a careful set up of methodologies and objectives.

In a successive analysis about the word "integration" [of history in mathematics teaching] Furinghetti (2002) supports the idea of using history as a mediator to pursue the objectives of mathematics education. This means to develop an analysis of these objectives together with the study of the development of concepts in history. Such work has to be carried out by educators and historians in a collaborative way: examples involving infinitesimal analysis are given. According to this new perspective, the question about the symmetry of the two roles (played by historians and didacticians) is no longer of interest: the role of history in teaching mathematics requires proper analysis and proper competence.

At this regard, interesting examples are given by Vinner (1988) and Tirosh and Almog (1989) on students' understanding of complex numbers, and by Sfard 1991, on the concept of function . 


\section{A Second Case: Mathematics and Didactics of Mathematics}

The education of the researchers in didactics of mathematics is different in different countries. In some countries they are educators with a further specialization in mathematics; in some others they are mathematicians with a further specialization in didactics. The relationships between the two communities (mathematicians and researchers in Mathematics Education) are not always easy, not even in those countries where didacticians are a subset of mathematicians. It may happen that studies in Mathematics Education are scarsely (or even not) recognised as valuable scientific research.

Sometimes the disagreement between mathematicians and didacticians of mathematics takes extremely visible forms. An example for this comes from the United States, i. e. the hot on-line debate circulated by Jerry Becker < jbecker@siu.edu> starting from the open letter, signed by a group of mathematicians and scientists to USA Secretary of Education, Richard Riley, published in the Washington Post, November 18, 1999, and then the reactions. Roughly speaking, mathematicians and scientists consider themselves to be the guardians of the theoretical nature of mathematics, whilst educators are supposed to be the supporters of the needs and difficulties of the teachers and of the learners. An outsider observer could say that we are looking at a role game where two communities of players are defending, we may hope in good faith, ideological positions. Hyman Bass, the president of ICMI, assumed a very balanced position and was aware that the publication of the Open Letter, might have had dangerous effects:

'The Open Letter is notable for featuring prominent mathematicians and scientists, and few, if any, teachers and educators who are knowledgeable about the curricula in question, and who know professionally what is involved in teaching in the schools. A possible subtext of this choice is the suggestion that these mathematicians and scientists are the final, and perhaps exclusive, intellectual authority on evaluating the quality of instructional materials in math and science. I am a mathematician who is interested in the improvement of mathematics education in this country, and I am deeply convinced that the expertise of my professional community has a vital role to play in educational research and policy. I have done my best to achieve such involvement. But I find that the implication symbolized by the list of signers of the Open Letter to be not only wrong, but dangerous and damaging. Ironically, it does a great deal to make serious professional collaboration impossible' (Hyman Bass, November, $27^{\text {th }}$ 1999)

However, what seems to be lacking in the discussion are the references to the great amount of research studies in the field of didactics of mathematics, which can give consistent orientation to curriculum development. In fact, most studies are concerned with the deep processes related to the 
are investigated. For this reasons, such studies could usefully ground the didactic choices underlying the curriculum profile.

The example of the open letter to Riley witnesses the difficulties of the dialogue between the two scientific communities of professional mathematicians and professional didacticians of mathematics. Some other misunderstandings are common. Recently in the most important international forum of mathematicians, The International Congress of Mathematicians, one of the parallel session was devoted to 'Teaching and popularization of mathematics' (1998) and "Mathematics Education and popularization" (2002). Some researchers in didactics of mathematics were invited as official lecturers (e. g. Artigue, 1998; Bartolini Bussi, 1998; Niss, 1998). However, why is teaching paired with popularization? Is it a way of emphasizing once again that, for mathematicians, teaching mathematics is similar to popularizing mathematics? Whichever is the mathematicians' opinion, we must insist that they are related, yet different, things. Popularization might be related to only some aspects of the research in didactics of mathematics, like students' motivation and the reorganization of a field of knowledge around a few fundamental ideas. But teaching is much more (and much different) than popularisation: it takes new emphasis from the research findings in the specific field of Mathematics Education. Institutionally mathematicians should be interested in the teaching of mathematics, in order to educate further generations of mathematicians at least. So, we can ask the following question: Might the cooperation between didacticians and mathematicians be posed on a peer basis? In other words, might didacticians profit from the development of mathematics and, conversely, might mathematicians profit from the development of didactics of Mathematics?

The first question is not trivial. Of course didacticians are interested in the development of new mathematical ideas, because they are source and stimulus for their didactic transposition; but there is an additional contribution that mathematicians can offer. Explaining their ways of reasoning in solving and posing problems they can give useful hints to understand students' ways of thinking.

At this regard, we would also like to mention the sharp voice of one of the most important contemporary Italian mathematicians, Giovanni Prodi, to whom we owe much, for his cultural and political help and his personal involvement in the problems of teaching mathematics. The excerpt (translated by us) is taken from the concluding address to a national seminar on 'What is research in didactics of mathematics?' held in Pisa in 1991. 
[....] Despite the need of considering so many factors in mathematics education, I believe that there is a privileged sector for research, nearly isolated and favourable for mathematicians engaged in didactical research: I mean the psychology of cognitive processes. In fact, on the one hand, mathematical processes are among the clearest for the analysis of thinking and, on the other hand, the contribution of mathematicians to this field of enquiry is essential. (Prodi 1992).'

The second question (i.e. if mathematicians can profit from didacticians) is not trivial too.

In his seminal paper "On proof and progress in mathematics", William Thurston (1994) approaches the question "How do mathematicians advance human understanding of mathematics?", which is framed in the more general perspective on the ways to understand and think about mathematics. He recognises that there is a list of different ways of thinking about or conceiving of a concept (for example the cocept of derivative), rather than a list of different logical definitions.

Furthermore, he claims that "Human thinking and understanding do not work on a single track, like a computer with a single central processing unit. Our brains and minds seem to be organised into a variety of separate, powerful facilities. These facilities work together loosely, "talking" to each other at high levels rather than at low levels of organization"(ibidem, p.164). Later, he recognizes that the transfer of understanding from one person to another is not automatic, but "hard and tricky". "Therefore, to analyse human understanding of mathematics, it is important to consider who understand what, and when. Mathematicians have developed habits of communication that are often dysfunctional" (ibidem, p.165)

In this perspective, didactic research related to how we think about mathematics, how we write it and how we communicate understanding acquires basic importance. Consequently, mathematicians can highly profit from the work of didacticians: we strongly support this claim.

\section{Didactics of Mathematics and School Practice}

Until now we have analysed two cases of possible (not yet realized) dialogue and peer cooperation between communities of researchers, focusing on the relationships between didacticians (of mathematics) and historian (of mathematics) on the one hand and between didacticians and mathematicians on the other hand. We move now to another case of dialogue, that is supposed to be carried out at least in some particular situations, i.e. the dialogue between researchers in mathematics education and school practitioners. 
Theory and practice (i.e. the theoretical approach to the discipline and its transposition in school practice) has often been considered as opposite poles. In recent decades, the dialectic nature of the theory-practice relationship has become increasingly recognised and embodied in research studies, which have highly fostered the dialogue between the two poles (Brown and Cooney, 1991, Bazzini, 1991, Burton, 1991, Wittmann, 1991, Steinbring, 1994). In particular, a group of researchers started at ICME5 in Adelaide in 1984 a project named "Systematic Co-operation between Theory and Practice in Mathematics Education" (in short SCTP) expressly devoted to examine this problem field in terms of international co-operation. From the very beginning, an attempt was made to compare mathematics education with other specific fields in order to elaborate aspects of boundary conditions of mediating knowledge between the two poles. Following the conference in Adelaide, there was a continuing interest in the relationship between theory and practice in Mathematics Education which implied both practical efforts and theoretical reflection, as witnessed by the subsequent SCTP Conferences, held in Lochem (the Netherlands) in 1986, in London in 1988, in Brakel (Germany) in 1990 (see Seeger and Steinbring, 1992) and in Grado (Italy) in 1994 (see Bazzini, 1994), as well as the Topics Groups at ICME6 in Budapest (1988) and ICME7in Québec (1992).

It has been clearly stated that 'theory and practice can no longer be understood as competing to give the "real" picture of what goes on in the classroom:
they do reflect upon and speak about the same processes and structures, but with different voices, from different perspectives,
using different modes of reflection. What is needed, then, is the cooperation between theory and practice, is a dialogue where
the different voices are listening to each other' (Seeger, 1994). In short, any conception which assigns to "theory" the place of instructing "practice" is doomed to fail: a general consensus on the necessity of developing the notion of cooperation is widely shared. An interesting contribution is given by Ponte et al. (1994), when discussing a new type of professional knowledge for mathematics teachers. They support the idea of blending of mathematical content with pedagogical knowledge: in their view such blending has to draw on different components of current knowledge to produce a restructuring of the teachers craft knowledge. This pedagogical content knowledge (called "mathematical didactics" by the authors), has a much broader scope than just the representation of subject matter: it must include "a comprehensive body of images, principles, and rules for action, some general, some more specific, organized with a clear rationale, bearing on the specific nature of the underlying content and 
(1998) explores a specific component of professional knowledge for mathematics teachers, named "epistemological knowledge of mathematics in social learning settings". He claims that "teachers surely need mathematical content knowledge and pedagogical knowledge; and within the domain of pedagogical content knowledge, they also need epistemological knowledge so they are able to assess the epistemological constraints of mathematical knowledge in different social settings of teaching, learning, and communicating mathematics. This important component of epistemological knowledge of mathematics in social learning settings is not a systematized, canonical knowledge corpus which could be taught to future teachers in the way of a fixed curriculum. Rather, the epistemological knowledge consists of exemplary knowledge elements as it refers to case studies of

analysis of teaching episodes or of interviews with students, and comprises historical, philosophical, and epistemological conceptual ideas" (p.160). This notion is further investigated by the author with regards to the problematique of how mathematical signs and symbols gain meanings in the interactive social processes of teaching and learning and supports the claim that this kind of theoretical professional knowledge for mathematics teachers combines theoretical aspects with practical issues, but this does not imply a linear course from theory to practice. Rather, an interplay, a balance between theoretical and practical requirements is fostered: "Whereas the practical training often overemphasizes the practical needs in a way of ignoring the theoretical sensibility and also theoretical possibilities for the everyday teaching practice (often students are simply equipped with a repertoire of practical rules and traditional teaching methods void of any theoretical justification) the theoretical education must not separate theory and practice, but try to develop teacher students' theoretical abilities to look behind the practical surface problems they will encounter later in their teaching practice" (p. 187).

\section{Two examples of Projects Based on the Dialogue between Theory and Practice}

We now present, with some details, two cases of research projects, with a strong involvement of school teacher-researchers together with university researchers They concern the approach to theoretical thinking in geometry and the algebraic problem solving. In this presentation we do not deal with the results of the studies (for which only references to the literature are given), but rather with the effects on the studies of the dialogue between the needs of theoretical research in didactics 
First Example: The Approach to Theoretical Thinking in Geometry

In the early nineties research teams at the universities of Genoa (P. Boero), Modena (M. Bartolini Bussi), Pisa (M. A. Mariotti) and Torino (F. Arzarello) started to work independently on the problem of theoretical thinking in mathematics and proof.

The approach to theoretical thinking in mathematics is considered essential by the research team. The discipline 'Mathematics', as it has been developed in Western culture, is not an empirical discipline. The roots of Mathematics might well be in everyday and body (Lakoff \& Nunez) experience, yet the development of Mathematics brings to a specific systematization, where results are derived by deductive reasoning from some shared assumptions. In Mathematics the function of theorems and proofs is important, whilst in Mathematics education there had been a trend documented at the international level, to cancel theorems and proofs from mathematics curricula, as a reaction to the formal approach of the seventies (see the Curriculum and Evaluation Standards for School Mathematics (1989), Professional Standards for Teaching Mathematics (1991), and Assessment Standards for School Mathematics (1995)).

The research team considered essential to contrast this trend. However, saying that in Mathematics the deductive structure is important does not mean that Mathematics has to be taught from the very beginning in a deductive way. Even in defending the importance of proof, there is a difference between a mathematician's and a didactician's perspective.

\footnotetext{
'Research mathematicians often ignore the aspect of application and confine themselves to the purely deductive notion of proof. [...] Teachers, on the other hand, must take into consideration the contribution which a given proof makes to our understanding of reality. All those issues which the practicing mathematician can afford to ignore play an important role in teaching and in learning. Proof cannot be taught and learned without considering the relation of mathematics to reality. This implies a high level of epistemological complexity (Hanna and Jahnke, 1993, p. 432)'.
}

Exploratory studies were produced at different school levels (from primary to secondary school). The presence of the teachers was determinant in each phase ( design, implementation, collection of data and analysis). Their sensitivity and competence proved to be essential not only in the careful management of classroom activity but also in the elaboration of analytical tools and of the theoretical framework in didactics of mathematics. Last but not least, while taking part in the design of experiments, the teachers were put in the condition of deepening some issues concerning the theoretical dimension of mathematics and its relationship with experiential reality. in other words, the theoretical dimension of mathematics became part of the intellectual life of teachers. 
Most of teaching experiments developed in the project shared (and still share) some common features, from the design phase to the implementation in the classroom :

1) the selection, on the basis of historic-epistemological analysis, of fields of experience, rich in concrete and semantically pregnant referents (e. g. perspective drawing; sunshadows; Cabriconstructions; gears; linkages and drawing instruments);

2) the design of tasks within each field of experience, which require the students to take part in the whole process of production of conjectures, of construction of proofs and of generation of theoretical organisation;

3) the use of a variety of classroom organisations (e. g. individual problem solving, small group work, classroom discussion orchestrated by the teacher, lectures);

4) the encouragement of all the kinds of 'dirty' editing (i.e. drawings, numerical trials) of students' ideas and processes which come before the final clean text (i.e. what the student condiders his final version to be submitted to the teacher),

5) the explicit introduction of primary sources from the history of mathematics into the classroom at any school level.

Surely each of this feature may be shared by other approaches. The choice of activity with concrete referents, for instance, is typical of the 'Realistic Maths Education' approach, developed in Utrecht by the followers of Freudenthal ( Presmeg, 2001). The focus on either small group or whole class discussion is shared by the approach developed by Yackel \& Cobb (1996). The introduction of historical sources in the mathematics classroom is a common practice in (at least) a small group of researchers and practioners in mathematics education (see for instance the chapters 5, 7,9 and 10 in Fauvel \& van Maanen, 2000). What seem to us original are the following:

- the major focus on the theoretical dimension of mathematics (mainly development of theorems and proofs)

- the framing of such experiments in a theoretical framework, based on Vygotskij's ideas, where each of the elements is put in relation with the others.

According to a legacy of the Italian tradition, attention was paid initially to geometry theorems. Some theoretical constructs developed earlier were assumed by all the teams, implicitly or explicitly, to help in the design, the implementation and the analysis of classroom experiments, whose aim was to create the conditions for most of pupils to be able to produce proofs (Bartolini 
activity (see the construct of field of experience by Boero, Mariotti et al., 1997 ) and the quality of classroom interaction (see the construct of mathematical discussion) (Mariotti et al., 1997).

The outcomes of the teaching experiments were astonishing, if compared with the general plea about the difficulty (or the impossibility) of coping with the theoretical dimension of mathematics: most of students even in compulsory education (e. g. grades 5-8) succeeded in producing conjectures and constructing proofs. Had these studies been action research based, the process could have stopped here with the production and documentation of facts, i. e. paradigmatic examples of improvement in mathematics teaching. But the second aim of 'research for innovation' concerned the study of the conditions for realisation as well as the possible factors underlying effectiveness; in other words, this success had to be treated as a didactic phenomenon and brought up to theoretical clarification..

This created the need of enclosing, in an explicit way, the existing studies within a theoretical framework, where other theoretical constructs have been added to the two mentioned above ('field of experience' and 'mathematical discussion'). Such theoretical constructs might interpret the existing research studies in an integrated way and might suggest issues for a research agenda (Mariotti et al., 1997). Two specific exemplary elements of the theoretical framework are described below.

On the base of historic - epistemological analysis, a mathematical theorem is conceived as the system of three interrelated elements: a statement (i.e. the conjecture produced through experiments and argumentations), a proof (i.e. the special case of argumentation that is accepted by the mathematical community) and a reference theory (that includes not only postulates but also deduction rules - i.e. metatheory); this conception emphasises the importance that students are confronted with this complexity rather than with the mechanical repetition of given proofs. The cognitive unity (Pedemonte, 2002) is meant as the continuity between the processes of conjecture production and proof construction, recognisable in the close correspondence between the nature and the objects of the mental activities involved. The cognitive unity is the cognitive counterpart of a process recognizable in the activity of most mathematicians (Thurston, 1994) and used also in the rational reconstruction of the development of pieces of mathematical theories (see for instance the dialectic process of constructing proof and refutations, Lakatos, 1976 ). In didactical research is, on the one side, a formidable tool for designing activities within the reach of students and, on the other 
and failure. Later, this theoretical framework has been applied elsewhere and refined in order to be used to interpret and/or to predict the students' performances in specific cases. For instance, Arzarello (2000) has modelled the whole process of producing a conjecture and constructing a proof in the rich field of experience of Cabri constructions, with a fine grained analysis of several kinds of dragging. The detailed observations of the teacher-researchers in the classroom on the one hand have suggested new directions of research (such as the development of a theory of semiotic mediation, see Bartolini Bussi et al. 1999; Bartolini Bussi \& Mariotti, 1999; Mariotti \& Cerulli, 2002) and on the other hand have been interpreted by referring to the existing literature and to the new studies developed together with them.

\section{Second example: Algebraic Problem Solving}

The project on algebraic thinking we are going to describe is another example of close co-operation between academic researchers and school teachers. Roughly speaking, we can distinguish three phases, which involved the two components (academics and teachers) with different roles and expertice.

First phase

The project started from the observation by school teachers of students at work when facing algebraic problem solving (i.e. problems involving the use of algebraic expressions). In particular it was observed by teachers that many difficulties were connected with mastering symbols. The analysis of students' protocols showed that some troubles were rooted in the students' incompetence in constructing and mastering suitable formulas, which should incorporate the significance of the objects involved and their mutual relationships (Arzarello, Bazzini and Chiappini, 1993, 1994b, 1994c ). These basic considerations led to an extended discussion on the very nature of algebraic thinking and on the construction of meaning in algebra. Such a discussion involved different perspectives, strictly interconnected and related to psychological, epistemological and didactical aspects.

As a consequence, the need for theoretical investigation into the dynamics which underly algebraic thinking emerged in its whole complexity.

Second phase

The second phase was characterized by a theoretical reflection on algebraic thinking, which was 
kind of thinking, lives in the interplay between mental activity and linguistic expressions. In this perspective, the role of algebraic language is particularly important. More precisely we can say that, in general, representations and symbols of mathematics establish semiotic systems which are of fundamental importance in doing algebra.

In this framework, we have been concerned with a theoretical model apt to describe the dynamics of some typical processes.

The ideas held by Frege on semantics appeared suitable for looking at the interpretation of symbolic expressions of algebra (Arzarello, Bazzini and Chiappini, 1994a, 1994b, 1994c). The epistemological triangle by Frege (1892) was applied to algebraic expressions in order to distinguish between two basic components (sense and denotation) of an algebraic expression. Although we were conscious that Frege was not interested in the thinking of children, and he possibly would have rejected a psychological interpretation of his terms, the distinction between sense and denotation of a given algebraic expression seemed to be very useful in the investigation of algebraic thinking. According to these lines, we adapted the Frege's model to our purposes.

Following Frege's terminology, we distinguished between the sense and the denotation of an expression: the denotation of an expression is the object to which the expression refers, while the sense is the way in which the object is given to us.

We consider permitted algebraic transformations those which allow one to get different expressions which have a (possibly) different sense but maintain the same denotation. Hence, algebraic transformations ( like, for example, adding the same numer to both sides of an equation) are invariant with respect to the denotation of the symbolic expression they act upon, because they can change the sense but not the denotation of the symbolic expression itself. The converse is not true. If two different expressions have the same denotation, they are not always reducible to each other by algebraic transformations (for example the two equations $x_{-}+2=0$ and $x_{-}+5=0$, considered in $R$, have both the empty set as denotation. The dialectic between sense and denotation allows to consider algebraic reasoning as a game of interpretation: a given formula can activate different senses and symbols' manipulation is promoted by the passage from one sense to another. In short, doing algebra as a game of interpretation considers the interpretation of given formulas in terms of activated senses and the related transformations according to the goal of the problem.

Third phase 
The third phase is mainly concerned with a reflection on the teaching-learning process. As such, the two components involving university researchers and school teachers closely co-operate. There is a general consensus that cognitive processes, which develop in teaching/learning algebra, cannot be separated from the analysis of the specific cultural practices and specific symbolic tools used.

Theoretical reflection on the very nature of algebraic thinking puts light on some typical students' behavior. It seems that very often a sort of rigidity makes students act as if there were a one-to-one correspondence between sense and denotation. By identifying both, the former disappears and the student remains with just a trivial denotation: the symbolic expression denotes itself. Thus algebra becomes pure syntax and nothing more.

The model sketched above pictures algebraic difficulties as a deficiency in mastering the invariance of denotation with respect to sense (Bazzini, 1997, 1998). For example, pseudostructural students analyzed by Linchevsky and Sfard (1991) do not recognise, when facing equations, the invariance of the concept of truth set, i.e. the set of numbers which must not change under the 'permitted' operations. Since they do not see such an invariance, they become "formalist", in the sense that they reveal a basic inability to link algebraic rules to the laws of arithmetic and so formal manipulations remain as the only source of meaning .

On the other hand, good solvers show great flexibility in the activation and anticipation of different senses of a given formula (for example $x^{2}-4$ can be seen as the difference between $x^{2}$ and 4 and also as the result of $(x+2)(x-2))$ and in the passage from one sense to another.

The interplay between sense and denotation of a given algebraic expression, as described in the theoretical model, provides concrete suggestions for designing suitable didactic situations. (Arzarello, Bazzini and Chiappini, 1995).

In particular, some main features of a suitable planning of teaching activities have been identified. They are mainly oriented towards the following goals

(1) motivating pupils for their planning of the activity;

(2) supporting them in the choice of the specific goals and in the consequent processes of anticipation and planning;

(3) emphasising the functionality of the system of signs into which the knowledge to be acquired must be incorporated, according to the design of the teacher;

(4) supporting a mathematical discourse in the classroom 
In short, the relationships between external representation, mathematical discourse and mental images can be sketched in this way within a didactical activity. The structure of external representations mediates the possibility of developing a meaningful mathematical discourse about the properties of the mathematical object, starting from how the properties of the external representation are used. This is in accordance with most research findings related to the use of representations and symbols in doing mathematics (see, for example, Radford, 2000). What is different in our study is the construction of a theoretical tool (i.e. the adapted epistemological triangle) which helps in analysing the very nature of algebraic expressions and, consequently, to interpret students' strategies in problem solving.

\section{Concluding Remarks}

The two above research projects are in the core of what is called the Italian paradigm of research for innovation (Arzarello \& Bartolini Bussi 1998). The roots of these projects are in the trends of research in didactics of mathematics, present in Italy for many decades. In the quoted paper, the authors reconstructed these roots, identifying different research traditions: concept-based didactics; innovation in the classroom; laboratory observation of processes. The arguments of the quoted paper are briefly summed up in the following.

The first two trends began in two different places (the university / the school) and carried out by different communities (the mathematicians / the teachers of mathematics); addressed different objects (the teaching of Maths in 'generic' / 'specific' situations); answered different needs (to produce ideas for improving Maths teaching / to produce improvement in Maths teaching); adopted different methodologies (top-down model / action-research); focused on different problems (products / short and long term processes in Maths teaching); and offered different products (textbooks, tests, syllabuses / projects for curricular innovation). The third trend was typical of educational studies. It consisted in basic research studies about short term processes, developed around laboratory experiments (where the classroom itself could be used as a laboratory), with analytical tools borrowed from psychology, sociology, pedagogy and so on ; the methodology was based on experimental induction, as it is conceived in natural science.

In other countries (e. g. France and Germany, see Arzarello \& Bartolini Bussi , 1998, for a detailed discussion) in spite of the presence of similar traditions, the pressure of academic 
of theoretical research (e. g. 'concept based research' or 'laboratory observation of processes') and forms of action-research (closer to 'innovation in the classroom'). In Italy (as in some other Countries, like, for example, The Netherlands) a different pathway was followed, namely the progressive integration of both traditions.

The two quoted projects are examples of the outcomes of this integration

In both projects

1) a deep analysis of the nature of mathematics is the starting point of inquiry, as in 'concept based research';

2) a strong involvement of teachers (from school and university) working in their own classrooms is the condition to carry out the experimental part of the study, as in 'innovation in the classroom';

3) the modeling of individual and social processes is realised, as in 'laboratory observation of processes'.

The integration of these traditions is embodied in teacher-researchers, who cooperate with didacticians from the universities and with other experts (e. g. psychologists, cognitive scientists and so on) driven by the needs raised by their classroom practice. Within these contexts professional didacticians and teachers have realized a truly peer cooperation.

As in the case of cooperation with teachers, we believe in cooperation with historians and with mathematicians too. It is a pity that our interest is not always returned, as we have shown in the first part of this paper. We are still far from the 'beautiful friendship' hoped for by Anna Sfard (1998) as a 'reflexive (we would call it 'symmetrycal', rather) relationship in which the difference is respected and the diversity becomes the source of mutual gain'.

The analysis on where and how such dialogue might be promoted, where and how it might be fruitful and what might be the obstacles is in need of further investigation. In this perspective, how does one explain these varying levels of success in interdisciplinary work and what are the implications for development could be central focus for discussion.

\section{References}

Arzarello F., 2000, Inside and Outside: Spaces, times and language in proof production, Proc. $24^{\text {th }}$ PME Int.. Conf., Hiroshima (Japan) vol. 1, 23-38.

Arzarello F. \& Bartolini Bussi M. G. 1998, Italian Trends in Research in Mathematics Education: A National Case Study in the International Perspective, in J. Kilpatrick \& A. Sierpinska (eds.), Mathematics Education as a 
Arzarello F., L.Bazzini, G.Chiappini, 1993, Cognitive processes in algebraic thinking: towards a theoretical framework, Proceedings PME-XVII, Tsukuba, Japan, Vol.I, 138-145.

Arzarello F. , L.Bazzini, G.Chiappini, 1994a, 'The process of naming in algebraic thinking', Proceedings PMEXVIII, Lisboa, Portugal, Vol.I,.40-47.

Arzarello F., Bazzini L., Chiappini G., 1994b Intensional semantics as a tool to analyze algebraic thinking, Rendiconti del Seminario Matematico, Univ. Torino. 52, N.2, 105-125.

Arzarello F., Bazzini L., Chiappini G.,1994c, L'Algebra come strumento di pensiero: analisi teorica e considerazioni didattiche, Progetto Strategico TID, Quad.N.6, Consiglio Nazionale delle Ricerche.

Arzarello F., Bazzini L., Chiappini G.,1995, The construction of algebraic knowledge:towards a socio-cultural theory and practice Proceedings of PME19, Recife, Brazil, Vol I, 119-134.

Bartolini Bussi M.,1996, Mathematical Discussion and Perspective Drawing in Primary School, Educational Studies in Mathematicss, 31 (1-2), 11-41.

Bartolini Bussi M. G.,1998a, Drawing Instruments : Theories and Practices from History to Didactics, Documenta Mathematica - Extra Volume ICM 1998, vol. 3, 735-746.

Bartolini Bussi M. G.,1998b, Italian Research in Innovation: Towards a New Paradigm, ICMI Bullettin, n. 45, $17-26$

Bartolini Bussi M. G. Mariotti M. A., 1999, Semiotic mediation : from history to mathematics classroom, For the Learning of Mathematics, 19 (2) 27-35

Bartolini Bussi M. G., Boni, M., Ferri, F. \& Garuti, R.,1999, Early Approach To Theoretical Thinking: Gears in Primary School, Educational Studies in Mathematics., 39 (1-3), 67-87.

Bazzini, L.,1991, Curriculum development as a meeting point for research and practice, Zentralblatt fuer Didaktik der Mathematik, 91/4, (128-131)

Bazzini L. (ed.), (1994) Theory and Practice in Mathematics Education, , Pavia, Italy, ISDAF.

Bazzini, L.: 1997, Equazioni e disequazioni: riflessioni sul concetto di equivalenza, in Bazzini L. (Ed.), La didattica dell'algebra nella scuola secondaria superiore, 44-53, Pavia, ISDAF.

Bazzini, L.:1998, On the construction and interpretation of symbolic expressions, in E. Cohors-Fresenborg, Proceedings of the First Conference on European Research in Mathematics Education, Osnabrueck.

Biehler R. , Scholz R. W., Straesser R. \& Winkelmann B.,1994, Didactics of Mathematics as a Scientific Discipline, Dordrecht, Kluwer Academic Publishers

Brown S., Cooney T., 1991, Stalking the Dualism between Theory and Practice, Zentralblatt fuer Didaktik der Mathematik 91/4, 112-117.

Burton L., 1991, Models of systematic co-operation between Theory and Practice, Zentralblatt fuer Didaktik der Mathematik 91/4, 118-121.

Cole, M., 1985, The zone of proximal development: where culture and cognition create each other, in Wertsch J.V. (ed.) Culture, communication, and cognition: a Vygotskian perspective, Cambridge University Press, 146161.

Fauvel J. \& van Maanen J. (eds.), 2000, History in Mathematics Education: the ICMI Study. Dordrecht, Kluwer Academic Publisher.

Frege, G., 1892, Über Sinn und Bedeutung, Zeitsch. für Philosophie und Philosophische Kritik, C, 25-50.

Furinghetti, F., 1997, 'History of mathematics, mathematics education, school practice: case studies linking different domains', For the Learning of Mathematics, 17, n.1, 55-61. 
Furinghetti, F., 2002, 'On the role of the history of mathematics in mathematics education', in I. Vakalis, D. Hughes Hallett, C. Kourouniotis, D. Quinney \& C. Tzanakis (eds), Proceedings of ITCM2 (Hersonissos, Crete, Greece), CD-Rom, J. Wiley \& Sons. 51.

Godino J.D. \& Batanero C. 1997, The dialectic relationship among theory, development and practice in mathematics education: a meta-analysis of three investigations, in N. A. Malara (ed.), An International View on Didactics of Mathematics as a Scientific Discipline, Proc. WG 25, Seville 1996), Università di Modena, Italy.

Godino, J.D., Batanero C.,1997, Clarifying the meaning of mathematical objects as a priority area of research in mathematics education, in A. Sierpinska and J. Kilpatrick (Eds.), Mathematics Education as a research domain: The search of an identity, 177-195, Dordrecht, Kluwer Academic Publishers.

Hanna G. \& Jahnke H. N. ,1993, Proof and Application, Educational Studies in Mathematics, 24, 421-438.

Hanna G. and Jahnke H. N., 1994, The Theory and Practice of Proof, in Gaulin C., Hodgson B. R., Wheeler D. H., Egsgard J. C. (eds.), Proceedings of the $7^{\text {th }}$ Intenational Congress on Mathematical Education, 253-256, Les Presses de l'Université Laval.

Horng W.-S., Lin F.-L. (Eds), 2000, Proceedings of the HPM 2000 Conference History in mathematics education. Challenges for a new millennium. A satellite meeting of ICME-9.

Inter-IREM Commission ,1997, History of mathematics histories of problems, Paris: Ellipses.

Inter-IREM Commission, 1998, Images, imaginaires, imaginations: une perspective historique pour l'introduction des nombres complezes, Paris: Ellipses.

Jahnke H. N., Knoche N. \& Otte M. (hrsg.), Geschichte der Mathematik in der Lehre,Goettingen: Vandenhoeck \& Ruprecht.

Kilpatrick J. \& Sierpinska A. (eds.), 1998, Mathematics Education as a Research Domain: A Search for Identity, Kluwer Academic Publishers.

Lagarto M. J., Vieira A. \& Veloso E. (eds.), 1996, Proceedings of the Second European summer university on 'History and epistemology in mathematics education' (Braga, Portugal), v.I, 261-262.

Lakatos I. ,1976, Proof and Refutations: the logic of mathematical discovery, Cambridge University Press

Lalande F., Jaboeuf F. \&. Nouazé Y. (eds), 1993, Proceedings of the First European summer university on 'History and epistemology in mathematics education' (Montpellier, 1993).

Linchevski L., Sfard A.: 1991, Rules without reasons as processes without objects. The case of equations and inequalities, in F. Furinghetti, Proceedings of the Fiftheenth PME Conference, Vol.II,.317.

Mariotti M. A., Bartolini Bussi M., Boero P., Ferri F. \& Garuti R., 1997, Approaching Geometry Theorems in Contexts: From History and Epistemology to Cognition, Proceedings of the $21^{\text {st }}$ PME Conference,1 180-195, Lahti, Finland.

Mariotti M.A., Cerulli M.,2002, L'Algebrista: a microworld for teaching and learning Algebra, Sciences et Techniques Éducatives, Numéro spécial algèbre vol 9 n¹-2, 2002

Pedemonte B.: 2002, Etude didactique et cognitive des rapports de l'argumentation et de la démonstration dans l'apprentissage des mathématiques These de dectorat, Université Joseph Fourier-Grenoble I, Università di Genova Pepe L.,1990, Storia e Didattica della Matematica, L'Educazione Matematica, vol. XI suplement to n. 2), $23-33$.

Ponte J., Matos J.F., Guimaraes Leal C. \& Canavarro A.P.,1994 Teacher's and students' view and attitudes towards a new mathematical curriculum: A case study. Educational Studies in Mathematics, 26, 347-365.

Presmeg N. (ed.) 2001, Realistic Mathematics Education Research: Leen Streefland's work continues, 
Prodi G.,1992, Ricerca in Didattica della Matematica, L'Insegnamento della Matematica e delle Scienze Integrate, 15 (10), 987-994.

Radelet, P.: 1999, Procedings of the Tthird European summer university on 'History and epistemology in mathematics education' Louvain La Neuve-Leuven.

Radford L., 2000, Signs and meanings in students' emergent algebraic thinking: A semiotic analysis, Educational studies in Mathematics, 42, (3) PAGINE

Seeger F.,1994, Co-operation between Theory and Practice in Mathematics Education, in Gaulin C., Hodgson B. R., Wheeler D. H., Egsgard J. C. (eds.), Proceedings of the $7^{\text {h }}$ Intenational Congress on Mathematical Education, 282-5, Les Presses de l'Université Laval.

Seeger F., Steinbring H. (eds.), 1992, The Dialogue between Theory and Practice in Mathematics Education; Overcoming the Broadcast metaphor, Materialien und Studien Band 38, IDM Bielefeld, Germany.

Sfard A. 1991, On the Dual Nature of Mathematical Conceptions: Reflections on Processes and Objects as Different Sides of the Same Coin, Educational Studies in Mathematics, 22, 1-36.

Sfard A., 1998, in Kilpatrick J. \& Sierpinska A. (Eds.), Mathematics Education as a Research Domain : A Search for Identity, vol. 2, 491-511, Dordrecht, Kluwer Academic Publishers.

Steinbring, H.,1994, Dialogue between Theory and Practice in Mathematics Education, in Biehler R., Scholz R. W., Straesser R. \& Winkelmann B., Didactics of Mathematics as a Scientific Discipline, Dordrecht Kluwer Academic Publishers.

Steinbring H.,1997, Epistemological constrints of mathematical knowledge in social learning settings. In J. Kilpatrick , A. Sierpinska (eds.) Mathematics Education as a research domain: A search for identity, Dordrect, Kluwer Academic Publishers.

Steinbring H.,1998, Elements of Epistemological Knowledge for Mathematics Teachers, Journal of Mathematics Teacher Education, 1, 157-189.

Steiner H. G.,1985, Theory of Mathematics Education: An Introduction, For the Learning of Mathematics, 5(2), 11-17.

Tirosh D., Almog N., 1989, Conceptual adjustments in progressing from real to complex numbers, Actes de la $13^{\wedge}$ Conférence Internationale Psychology of Mathematics Education, Paris, France, Vol. 3, 221-227.

Thurston W.P., 1994, On proof and progress in mathematics, Bullettin of the Americam Mathematical Society, 30, (2), 161-177.

Vinner S., 1988, Subordinate and superordinate accomodations, indissociability and the case of the complex numbers, International Journal of Mathematics Education and ScienceTechnology, 19, 593-606.

Wittmann E. Ch.,1991, From Inservice-Courses to Systematic Cooperation between Teachers and Researchers, Zentralblatt fuer Didaktik der Mathematik 91/5, 158-160.

Wittmann E. Ch., 1995, Mathematics Education as a "Design Science", Educational Studies in Mathematics, 29,355-374.

Yackel E., Cobb P. (1996). Sociomathematical norms, argumentation and autonomy in Mathematics, Journal for Research in Mathematics Education, 22 (390-408). 
Maria G. Bartolini Bussi

Dipartimento di Matematica

Università di Modena e Reggio Emilia

Via Campi 213 b

41100 Modena, Italy_bartolini@unimore.it

\author{
Luciana Bazzini \\ Dipartimento di Matematica \\ Università di Torino \\ Via Carlo Alberto, 10
}

10123 Torino, Italy_luciana.bazzini@unito.it 